\title{
Soluble SorCS1 binds the insulin receptor to enhance insulin sensitivity
}

\author{
Mads Kjolby \\ Aarhus University https://orcid.org/0000-0002-1043-6137 \\ Peter Breining \\ Aarhus University \\ Karen Pedersen \\ Aarhus University \\ Niels Wellner \\ Aarhus University \\ Karen Sørensen \\ Aarhus University \\ Anette Gjessing \\ Novo Nordisk Foundation Center for Basic Metabolic Research \\ Dovile Januliene \\ Osnabrück University
}

Dorthe Vittrup

Aarhus University

Arulmani Manavalan

Department of Neuroscience, Mayo Clinic

Maarten Rotman

Department of Neuroscience, Mayo Clinic

Gangadaar Thotakura

Department of Neuroscience, Mayo Clinic

\section{Guido Hermey}

Hamburg University

Peder Madsen

Aarhus University

Christian Vaegter

Aarhus University

Søren Thirup

Aarhus University

Olof Asplund

Lund University Diabetes Centre

Rashmi Prasad 
Lund University https://orcid.org/0000-0002-4400-6741

\section{Edwin Parlevliet}

Dept. Medicine, Div. Endocrinology, and Einthoven Laboratory for Experimental Vascular Medicine, Leiden University Medical Center (LUMC)

\section{Patrick Rensen}

Leiden University Medical Center https://orcid.org/0000-0002-8455-4988

\section{Andreas Madsen}

RMPC, Department of Biomedical Sciences, Copenhagen University, Denmark

\section{Birgitte Holst}

University of Copenhagen https://orcid.org/0000-0001-7432-097X

\section{Olav Andersen}

Aarhus University

\section{Arne Moller}

Osnabrück University

\section{Torben Hansen}

University of Copenhagen https://orcid.org/0000-0001-8748-3831

Anders Nykjaer ( $\boldsymbol{D}$ an@biomed.au.dk )

Aarhus University

\section{Article}

\section{Keywords:}

Posted Date: January 28th, 2022

DOI: https://doi.org/10.21203/rs.3.rs-1299440/v1

License: (c) (1) This work is licensed under a Creative Commons Attribution 4.0 International License. Read Full License 


\section{Soluble SorCS1 binds the insulin receptor to enhance insulin sensitivity.}

Mads Kjolby ${ }^{1,2,3^{*}}$, Peter Breining ${ }^{1,2,3^{*}}$, Karen Marie Pedersen ${ }^{1,3}$, Niels Wellner ${ }^{1,3}$, Karen Marie Juul Sørensen ${ }^{1,3,4}$, Anette Marianne Prior Gjesing ${ }^{5}$, Dovile Januliene ${ }^{4,8}$, Dorthe Maria Vittrup ${ }^{3}$, Arulmani Manavalan ${ }^{6}$, Maarten Rotman ${ }^{6}$, Gangadaar Thotakura ${ }^{6}$, Guido Hermey ${ }^{7}$, Peder Madsen ${ }^{3}$, Christian B. Vaegter $^{3}$, Søren Thirup ${ }^{4}$, Olof Asplund ${ }^{9}$, Rashmi B. Prasad ${ }^{9}$, Edwin T. Parlevliet ${ }^{10}$, Patrick C.N. Rensen $^{10}$, Andreas Nygaard Madsen ${ }^{11}$, Birgitte Holst ${ }^{11}$, Olav M. Andersen ${ }^{1,3}$, Arne Möller ${ }^{4,8}$, Torben Hansen $^{5}$, Anders Nykjaer ${ }^{1,3,6 \#}$.

${ }^{1}$ PROMEMO and DANDRITE, Aarhus University, Denmark; ${ }^{2}$ Department of Clinical Pharmacology, Aarhus University Hospital, Denmark and Danish Diabetes Academy; ${ }^{3}$ Department of Biomedicine, Aarhus University, Denmark; ${ }^{4}$ Department of Molecular Biology, Aarhus University; ${ }^{5}$ Novo Nordisk Foundation Center for Basic Metabolic Research, Copenhagen University, Denmark; ${ }^{6}$ Department of Neuroscience, Mayo Clinic, Florida, USA; ${ }^{7}$ Institute for Molecular and Cellular Cognition, Center for Molecular Neurobiology Hamburg, University Medical Center Hamburg-Eppendorf; ${ }^{8}$ Osnabrück University, Frankfurt Am Main Area, Germany; ${ }^{9}$ Lund University Diabetes Centre, Malmö, Sweden; ${ }^{10}$ Dept. Medicine, Div. Endocrinology, and Einthoven Laboratory for Experimental Vascular Medicine, Leiden University Medical Center (LUMC), Leiden, The Netherlands; ${ }^{11}$ RMPC, Department of Biomedical Sciences, Copenhagen University, Denmark;

* Shared first authors. \#Corresponding authors 


$\begin{array}{ll}\text { Anders Nykjaer } & \text { Mads Kjolby } \\ \text { PROMEMO and DANDRITE } & \text { DANDRITE } \\ \text { Department of Biomedicine } & \text { Department of Biomedicine, } \\ \text { Hoegh-Guldbergsgade 10, } & \text { Hoegh-Guldbergsgade 10, } \\ \text { Aarhus University } & \text { Aarhus University } \\ \text { DK-8000 Aarhus C } & \text { DK-8000 Aarhus C } \\ \text { Denmark } & \text { Denmark } \\ \text { Phone: }+45 \text { 2899 2384 } & \text { Phone: +45 6086 6653 } \\ \text { Email:an@biomed.au.dk } & \text { Kjolby@biomed.au.dk }\end{array}$

Word count: 2511 words, including abstract (150), but excluding title, authors, figure legends and references. 
1 Type 2 diabetes has reached endemic proportions and is a substantial burden for the affected 2 patients and the society. Along with lifestyle factors, a number of genetic loci predisposing to type

32 diabetes have been identified, including SORCS1 that encodes the transmembrane receptor 4 SorCS1. The ectodomain of SorCS1 (sol-SorCS1) is shed from plasma membranes but the 5 biological function of this fragment is unknown. Here we show that sol-SorCS1 acts as a high6 affinity binding partner for the insulin receptor to stabilize the receptor and increase insulin 7 affinity, protein kinase $B$ activation, and glucose uptake in myocytes. Sol-SorCS1 is liberated from 8 adipocytes, and in diabetic patients the plasma concentration positively correlates with body mass 9 index, but inversely with plasma glucose. In mouse models of insulin resistance, exogenous sol10 SorCS1 restored insulin sensitivity. We conclude that sol-SorCS1 increases peripheral insulin 11 sensitivity and propose sol-SorCS1 as a novel insulin sensitizing adipokine and potential 12 antidiabetic agent. 
13 The mechanisms controlling insulin sensitivity and glucose metabolism are fundamental to all vertebrates. Insulin resistance is an early hallmark for the development of type 2 diabetes ${ }^{1,2}$ as insulin sensitivity and peripheral tissue glucose uptake is essential to acutely buffer plasma glucose. The downstream signaling pathways of the insulin receptor (IR) in skeletal muscle are well described ${ }^{3}$. Among the second messengers, the most prominent pathway regulating the insertion of GLUT4containing vesicles is the protein kinase B (AKT) signaling cascade ${ }^{3}$. However, whether this pathway can be dynamically regulated through interaction with co-receptors or other membrane proteins is unknown ${ }^{4-6}$. The existence of a molecular thermostat to adapt insulin sensitivity to the metabolic state would transform our conceptual understanding on how key metabolic processes can be controlled.

SorCS1 is a member of the Vps10p-domain family of sorting receptors that also comprises sortilin, SorLA, SorCS2, and SorCS3 ${ }^{7-10}$. Several studies have identified SORCS1 as a susceptibility locus for type 2 diabetes mellitus that is conserved between humans ${ }^{11-13}$, rats $^{14}$ and mice ${ }^{15}$. Recently, intracellular SorCS1 was shown to facilitate the biogenesis of insulin containing secretory granules, but a function outside the $\beta$-cells was not studied ${ }^{16}$. We therefore performed RNA sequencing on human samples focusing on major peripheral tissues involved in glucose homeostasis (Fig 1,a). Notably, we found that SorCS1 expression was highest in adipose tissue surpassing that of the pancreatic islets whereas expression in the liver was hardly detected. We previously showed that every hour up to $95 \%$ of SorCS1 (sol-SorCS1) is liberated from the plasma membrane by ADAM17/TACE mediated cleavage but the biological relevance of this observation is unknown ${ }^{17,18}$. Given that human and murine adipose tissue express ADAM17/TACE ${ }^{17}$, we examined, whether body mass index ( BMI) associates with the plasma concentration of sol-SorCS1. To this end we assessed the correlation between BMI and the concentration of sol-SorCS1 in plasma in a cohort of diabetic patients and healthy controls. We found 
that sol-SorCS1 positively correlated with BMI and most prominently did so in the diabetic cohort (Fig

1,b). Accordingly, sol-SorCS1 could be liberated from human adipocytes as it was clearly detected in the conditioned medium from human subcutaneous adipose tissue explants (Fig. 1,c). By immunoprecipitation (IP) we also detected SorCS1 expression in lysates of visceral fat from mice and differentiated murine 3T3-L1 adipocytes, respectively (Fig. 1,d-e). Notably, the conditioned medium of the 3T3-L1 cultures was enriched in the soluble form of SorCS1 that migrated slightly faster than the

full-length receptor present in the corresponding lysate. Although SorCS1 protein was found in the myocyte-derived murine cell line $\mathrm{C} 2 \mathrm{C} 12$ (Extended data 1,a), we failed to detect the soluble receptor in the conditioned media (Extended data 1,b). We next examined whether sol-SorCS1 levels is associated with plasma glucose. Remarkably, among the diabetic patients sol-SorCS1 inversely

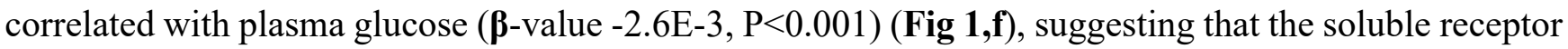
may impact glucose metabolism.

We hypothesized that sol-SorCS1 could serve as an adipose tissue derived peptide hormone to compensate for an increase in BMI by lowering plasma glucose levels and preventing hyperglycemia. To test this hypothesis, we sought to assess a potential glycemic regulatory function of the soluble receptor. We therefore injected an adenovirus encoding human sol-SorCS1 (AV-sol-SorCS1) or AVLacZ (encoding $\beta$-galactosidase), as a negative control, into the tail vein of diet-induced obese (39.5 $\pm 1.2 \mathrm{~g})$ hyperglycemic C57BL/6 mice (DIO mice). Eleven days after viral transduction, overexpression of the soluble receptor substantially mitigated the diabetic phenotype by lowering both the elevated plasma glucose and insulin levels corresponding to a $24 \%$ decrease in plasma glucose and a $22 \%$ reduction in insulin levels, respectively (Fig $\mathbf{2}, \mathbf{a}-\mathbf{b})$. To test the antidiabetic efficacy of sol-SorCS1 in another and more severe diabetic model, we turned to the leptin receptor knockout mouse model $(d b / d b)$ that rapidly develops progressive obesity and type 2 diabetes. One week after injection with AV- 
LacZ, plasma glucose was increased by $55 \%$ and insulin by $65 \%$ due to the gradual worsening of the diabetic phenotype (Fig. 2,c-d). Remarkably, following AV-sol-SorCS1 treatment plasma glucose remained unchanged and this despite a decrease in plasma insulin levels, indicating increased insulin sensitivity. In the intraperitoneal glucose tolerance test (IPGTT), AV-sol-SorCS1 mice responded markedly stronger with lower plasma glucose levels at all timepoints corresponding to a reduction in the area under the curve (AUC) by $79.5 \%$ (Fig. 2,e-f). The treated animals also exhibited superior ability in mobilizing insulin compared to the control group, revealing a spare capacity of insulin production that the control animals did not possess (Fig. 2g). Notably, regardless the level of sol-SorCS1 overexpression we never observed any signs of hypoglycemia due to a compensatory decrease in insulin secretion.

Next, we performed hyperinsulinemic euglycemic clamp ${ }^{19,20}$ which is commonly used to assess glucose deposition rates and insulin sensitivity. With the mice clamped to their basal fasting glucose levels (Fig. 2,h), the necessary glucose infusion rate to maintain stable plasma glucose levels in AV-sol-SorCS1 mice was substantially higher compared to the AV-LacZ group (Fig. 2,i). Notably, solSorCS1 increased peripheral glucose uptake by $41 \%$, demonstrating that sol-SorCS1 greatly increases insulin sensitivity in peripheral tissues (Fig. 2,j). The difference in severity of diabetes progression was further emphasized when measuring glycated hemoglobin (HbA1c) three weeks after viral injection in another set of mice (Fig 2,k). In the AV-LacZ group, HbA1c had increased considerably whereas it was only marginally higher in the AV-sol-SorCS1 group, indicating a long-lasting beneficial effect of the soluble receptor in reducing plasma glucose.

We previously reported that in neurons, the SorCS1 paralogues sortilin and SorCS2, can physically interact through their extracellular domains with the tropomyosin receptor kinase B (TrkB) that binds brain-derived neurotrophic factor (BDNF), to enable its signaling capacity ${ }^{21,22}$. We 
81 the genetic association with type 2 diabetes and its glucose lowering function, we asked whether this 82 tyrosine kinase receptor could be the insulin receptor. To assess this, we first performed co-IP experiments between SorCS1 and IR in transfected HEK293 cells in the absence or presence of insulin (Fig. 3,a). Antibodies against IR co-precipitated SorCS1 and vice versa, and this interaction was unaffected by preincubation with $100 \mathrm{nM}$ insulin, a concentration that saturates IR. Next, we evaluated the affinity of the receptor-receptor interaction by surface plasmon resonance (SPR) analysis using the extracellular domains of SorCS1, corresponding to sol-SorCS1, and the extracellular domain of IR. We found that sol-SorCS1 bound immobilized IR with an estimated affinity of $5 \mathrm{nM}$ (Fig. 3,b). Importantly, the interaction between SorCS1 and IR was not prevented by insulin, as the signals for binding of SorCS1 and insulin to IR separately were additive when the two ligands were co-injected (Fig. 3,c). Since insulin did not bind to immobilized SorCS1 (Extended Data 2), the combined data indicate that IR can form a trimeric complex with insulin and soluble SorCS1. Pro-insulin, glucagon, or glucagon-like peptide 1 (GLP-1) also did not bind to immobilized SorCS1 (Extended Data 2). We then asked whether full-length SorCS1, when embedded in plasma membrane, may regulate insulin binding to IR and its signaling capacity. First, we overexpressed IR in HEK293 cells (HEK-IR) alone or in combination with SorCS1$\mathrm{b}$, an isoform of SorCS1 that predominates at the plasma membrane ${ }^{18}$. Remarkably, SorCS1 increased the affinity of insulin to IR by approximately five-fold (Fig. 3,d). Given that overexpression of SorCS1 and IR could potentially result in ligand independent autoactivation, we compared insulin signaling in $\mathrm{C} 2 \mathrm{C} 12$ cells with or without CRISPR/Cas9 mediated knockout of Sorcs 1 . The most prominent pathway regulating the insertion of GLUT4 containing vesicles is the AKT signaling cascade. Indeed, we found that SorCS1-deficient $\mathrm{C} 2 \mathrm{C} 12$ cells were substantially less insulin responsive than control cells as the $\mathrm{EC}_{50}$ for pAKT was left-shifted approximately five-fold (Fig. 3,e and Extended Data 1,b). Together the data suggested that the glucose lowering effect of SorCS1 is accounted for by an allosteric change in IR 
104 that increases its insulin binding affinity, leading to stronger AKT signaling. To substantiate such a model, we measured the thermal stability of full-length IR in the absence or presence of sol-SorCS1 using a cellular thermal shift assay $(\text { CETSA })^{23}$. We found that incubation with sol-SorCS1 right-shifted the melting curve of IR in overexpressing HEK293 cells corresponding to an increase in $\mathrm{Tm}$ from $47.8^{\circ} \mathrm{C}$ to $49.5^{\circ} \mathrm{C}$ (Fig 3,f). The data suggests that sol-SorCS1 induces a conformational change in IR, and supports that allosteric changes in IR may be accountable for the beneficial effects of SorCS1 on insulin binding and signaling.

We previously reported that SorCS1 exists as a monomer and as a non-covalent dimer, with

112 the oligomeric state, being regulated by the level of glycosylation ${ }^{24}$. However, the functional implications

113 of the two forms, e.g. whether the relative amounts of the two forms dictate binding specificity remains

114 unknown. To explore the spatial distribution of the monomer and dimer at the subcellular level, we used

115 a bimolecular fluorescence complementation (BiFC) assay ${ }^{25}$. HEK-IR cells were transfected with two

116 human SorCS1 constructs each containing one-half of the Venus fluorescent protein. When SorCS1

117 dimerizes the two Venus fragments unite to elicit a green fluorescent signal that echoes the subcellular

118 distribution of dimeric SorCS1. First, we stained the cells with an antibody that recognizes both 119 monomeric and dimeric SorCS1 and found monomeric SorCS1 is present at the plasma membrane 120 whereas the BiFC-positive signal representing dimeric SorCS1 is restricted to intracellular compartments 121 (Fig. 4,a). This is in accordance with our previous observation demonstrating that through its maturation, 122 N-linked oligosaccharides transforms SorCS1 from the dimeric form into the monomeric configuration ${ }^{24}$.

123 Binding of insulin to the IR changes its dimeric and symmetric conformation to adapt an asymmetric 124 configuration (personal communication, Dr Poul Nissen, manuscript to be resubmitted). This prompted 125 us to study whether the interaction of IR is restricted to the monomeric form of SorCS1. To demonstrate 126 that monomeric SorCS1 and IR can interact, we first combined the BiFC signal with proximity ligation 
127 assay (PLA) for the two receptors. In this assay a red fluorescent signal appears when SorCS1 and IR are 128 in close proximity $(<40 \mathrm{~nm})$. We observed a strong PLA signal at the plasma membrane that was BiFC129 negative, indicating that only monomeric SorCS1 binds IR, which suggest that the biologically active 130 form of SorCS1 is the monomer (Fig. 4,b). To substantiate this hypothesis, we produced an adenovirus 131 encoding a truncated version of SorCS1 that is unable to dimerize and studied its biological activity.

132 SorCS1 harbors a consensus sequence (amino acids 695 to 702) for cleavage by prohormone convertases 133 of the subtilisin/Kex2-like family, such as furin ${ }^{26}$. Truncation at this site gives rise to a soluble fragment 134 of roughly $60 \mathrm{kDa}$ (aa130-695 after cleavage of the propeptide and signal peptide) that comprises the 135 entire Vps10p domain but does not possess the dimerization site (Extended Data 3). We overexpressed 136 this explicit monomer by adenovirus (AV-aa1-695-SorCS1) and compared its glucose lowering capacity 137 to AV-sol-SorCS1 (aa1-1097) that comprises the entire ectodomain and likely represents a mixture of 138 monomer and dimer. At day 7 and 14 after viral injection both soluble SorCS1 variants were equally 139 potent in reducing plasma glucose (Fig. 4,c). This finding indicates that shed monomeric sol-SorCS1 140 plays an important role in the biological effects on peripheral glucose disposal.

To demonstrate that the beneficial properties of sol-SorCS1 was not secondary to 142 intrahepatic effects upon hepatic overexpression and to clearly isolate the actions of monomeric from 143 dimeric sol-SorCS1, we studied the glucose lowering effect of the two species upon exogenous 144 administration. In practice, the two forms were purified by size exclusion chromatography from medium 145 of cells expressing sol-SorCS1 and the two species injected intraperitoneally into $d b / d b$ mice at $10 \mathrm{hrs}$ 146 and $1 \mathrm{hr}$ prior to a glucose tolerance test. Intriguingly, monomeric sol-SorCS1 profoundly improved 147 glucose tolerance whereas the dimeric version showed no effect on glucose disposal (Fig. 4,d). The AUC 148 for plasma glucose in the glucose tolerance test was reduced by approximately $60 \%$ (from 1060 to 393 $\left.149 \mathrm{mM}^{*} \min , \mathrm{P}<0.05\right)$ in animals treated with monomeric sol-SorCS1 (Fig. 4,e). 
Aimed at demonstrating a direct effect of monomeric sol-SorCS1 on muscle cells, we

151 developed a real time in vitro assay with a 1 minute resolution in cellular glucose-uptake (Fig. 4,f-g,

152 Extended Data 4) ${ }^{27}$. Applying this highly sensitive real-time assay on primary murine myotubes,

153 revealed that sol-SorCS1 does not function as an insulin mimetic, since preincubation with either variant

154 of sol-SorCS1 for 20 minutes did not increase uptake of the glucose analogue (Extended Data 4,b).

155 Subjecting naïve myotubes to $10 \mathrm{nM}$ insulin had no effect on glucose analogue uptake. However, after

156 preincubation with $100 \mathrm{nM}$ monomeric sol-SorCS1 addition of $10 \mathrm{nM}$ insulin led to a $45 \%$ increase in

157 glucose uptake rate (Fig $\mathbf{4 , h}-\mathbf{i})$. In marked contrast, $10 \mathrm{nM}$ insulin stimulation after preincubation with

158 dimeric sol-SorCS1 which showed no such effect. However, addition of $100 \mathrm{nM}$ insulin to naïve or

159 dimeric sol-SorCS1 treated myotubes increased glucose uptake to match the rate obtained for myotubes

160 stimulated with $10 \mathrm{nM}$ insulin and pretreated with the sol-SorCS1 monomer (Extended data 4,c). Hence,

161 monomeric sol-SorCS1 acts directly on myotubes to enhance their insulin sensitivity and induces a rapid

162 increase in glucose uptake.

163

In conclusion, we have demonstrated that SorCS1 circulates in plasma in a soluble form

164 that can be produced by adipose tissue. Once secreted it acts as a hormone that targets tissues remote

165 from its site of production to increase insulin sensitivity. Accordingly, overexpression of the soluble

166 receptor fragment reduces plasma glucose and insulin levels in mice with diet induced obesity and limits

167 diabetes progression in leptin receptor knockout mice. We provide evidence that the biological activity

168 of sol-SorCS1 is restricted to its monomeric form and suggest that once liberated from the plasma

169 membrane it acts in an endocrine fashion (Extended Data Fig. 5). Our study nominates sol-SorCS1 as

170 a novel adipokine to control peripheral glucose metabolism and insulin sensitivity, and as a template for

171 future anti-diabetic drug development.

172 


\section{Acknowledgements}

175 The study was funded by the Lundbeck Foundation grant no. R248-2017-431 (AN), DANDRITE-R248-

176 2016-2518 (AN), R90-2011-7723 (AN); The Independent Research Fund Denmark grant no. DFF-7016-

17700261 (AN); The Novo Nordisk Foundation grant no. NNF16OC0020984 (AN); The Danish National 178 Research Foundation grant no. DNRF133 (AN); The Danish Diabetes Academy, Novo Nordisk 179 Foundation (MK), Lundbeck Foundation Emerge (MFK, AN). We thank Benedicte Vestergaard, Anja 180 Aagaard and Anne Kerstine Thomassen for excellent technical assistance and Professor Alan Attie, 181 University Wisconsin-Madison, WI, for insightful discussion.

182 
Figure 1

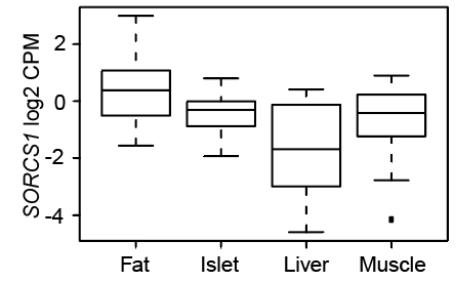

d Mouse visceral adipose

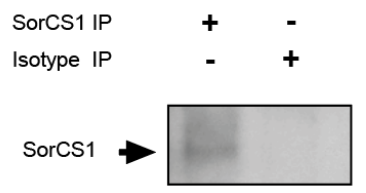

Correlation between

Sol-SorCS1 and BMI

b

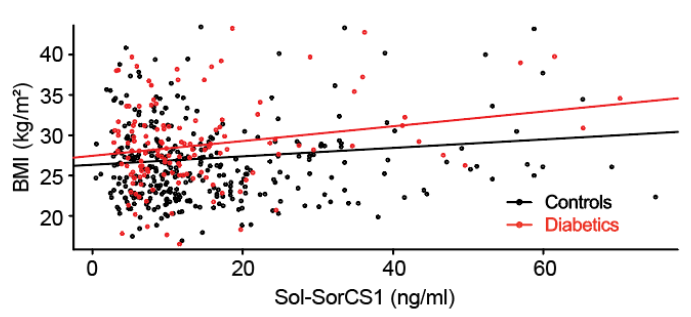

c

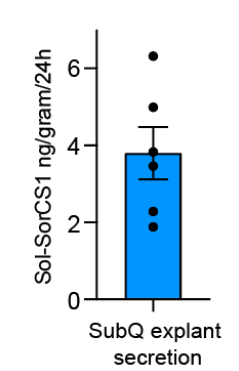

183

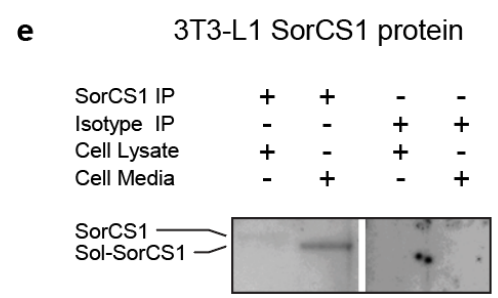

f Correlation between

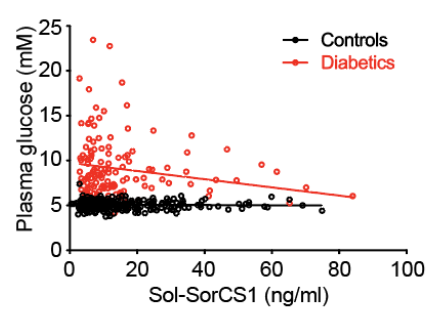

184 Figure 1: SorCS1 is expressed in, and secreted from, adipose tissue

185 a, mRNA expression of SORCS1 in human tissues ( $\mathrm{n}=12 /$ group). $\mathbf{b}$, in a group of healthy controls and

186 diabetes patients $(\mathrm{n}=328$ and $\mathrm{n}=124$, resp.) plasma levels of sol-SorCS1 positively correlate with BMI

187 ( $\beta$-value 0.011, $\mathrm{P}=0.007$ and $\boldsymbol{\beta}$-value 0.058, $\mathrm{P}<0.05$, respectively). $\mathbf{c}$, Sol-SorCS1 ELISA of media from

$18824 \mathrm{~h}$ incubation of human subcutaneous adipose tissue (SubQ) explants $(3.80 \pm 0.68 \mathrm{ng} / \mathrm{gram} / 24 \mathrm{~h})$. d,

189 protein immunoblot of murine SorCS1 in adipose tissue. e, full-length SorCS1 and its soluble shed form

190 in 3T3-L1 adipocytes. f, in the human cohort sol-SorCS1 negatively correlates with fasting plasma

191 glucose $(\boldsymbol{\beta}$-value $-2.6 \mathrm{E}-3, \mathrm{P}<0.001)$. 
Figure 2
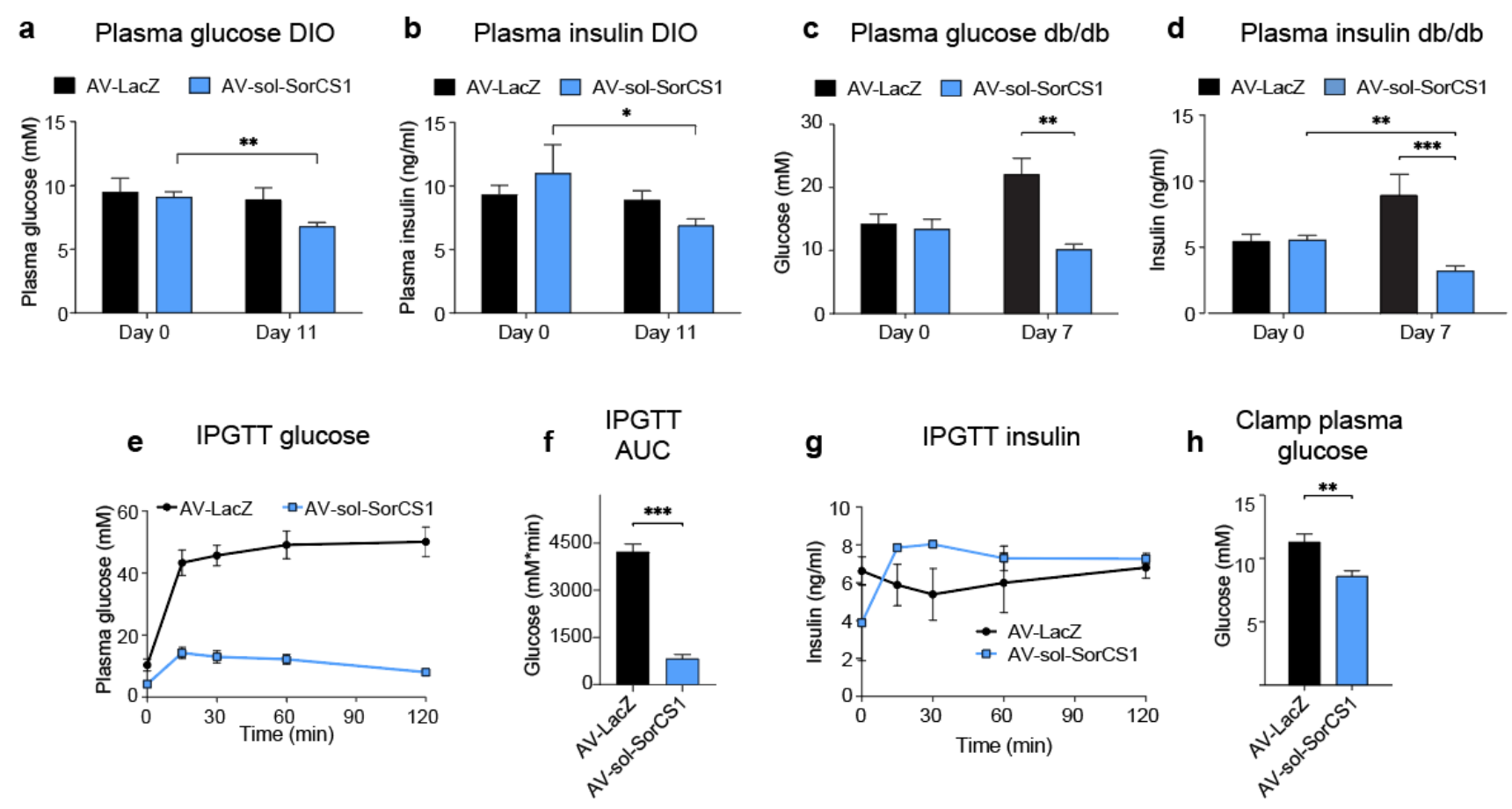

Clamp plasma
glucose
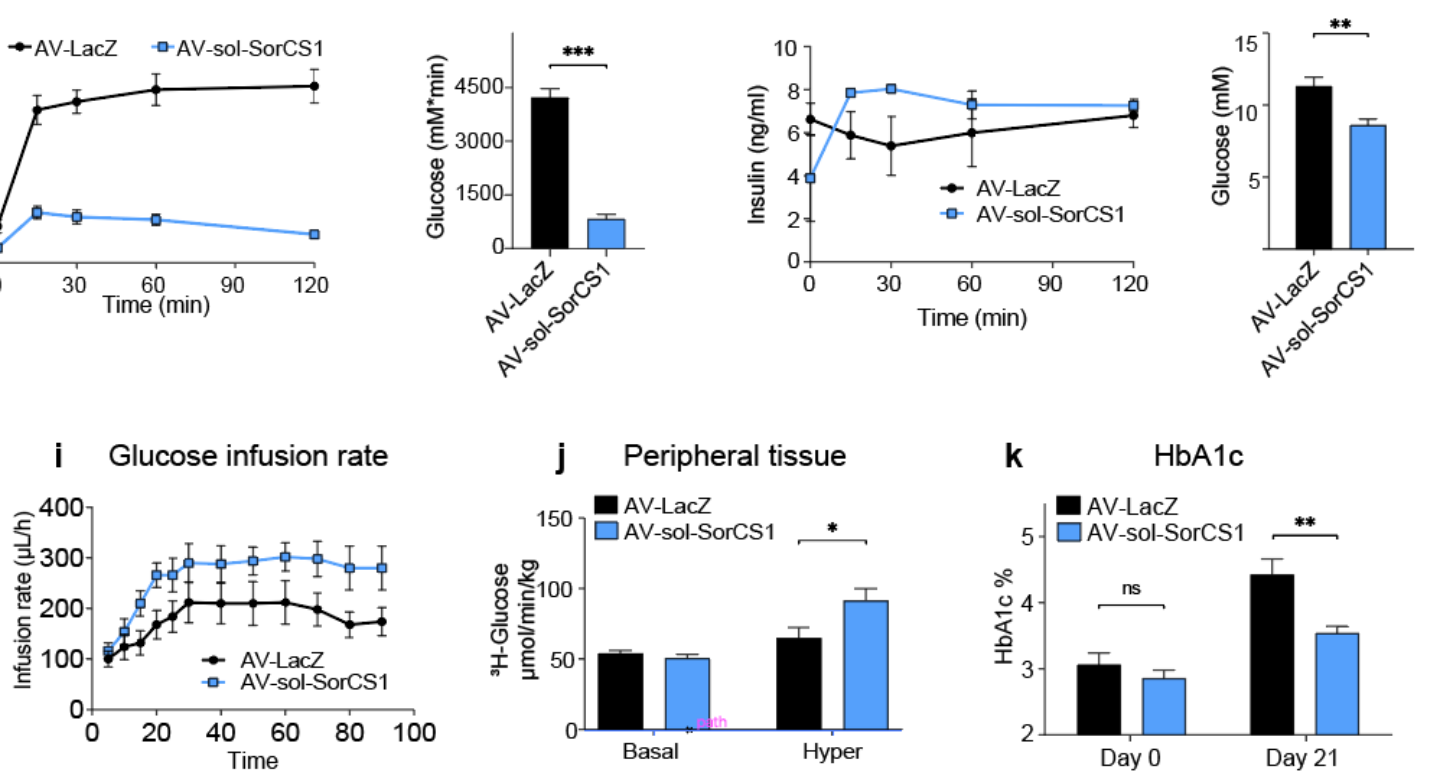

193 Figure 2: Sol-SorCS1 is an insulin sensitizer.

194 a-d, treatment with AV-sol-SorCS1, but not AV-LacZ, reduces plasma glucose levels and plasma insulin

195 in 16 weeks old diet-induced-obese (DIO) mice and in 7 weeks old $d b / d b$ mice. e, enhanced glucose 196 uptake determined by IPGTT in AV-sol-SorCS1 treated $d b / d b$ mice shown in real-time and, f, as AUC 197 (n=4/group). g, plasma insulin in the IPGTT shown in e-h. h, lower initial fasting plasma glucose in AV198 sol-SorCS1 treated $d b / d b$ mice but higher infusion rate during euglycemic hyperinsulinemic clamp, i 
199 ( $\mathrm{n}=5 /$ group). $\mathbf{j}$, the higher infusion rate is accounted for by increased peripheral glucose uptake (primarily 200 muscle tissue). $\mathbf{k}$, reduced HbA1c in $d b / d b$ mice 21 days after infection with AV-sol-SorCS1 infection.

201 Data are presented as means $\pm \mathrm{SEM}, *=P<0.05, * *=P<0.01, * * *=P<0.001$. 
Figure 3
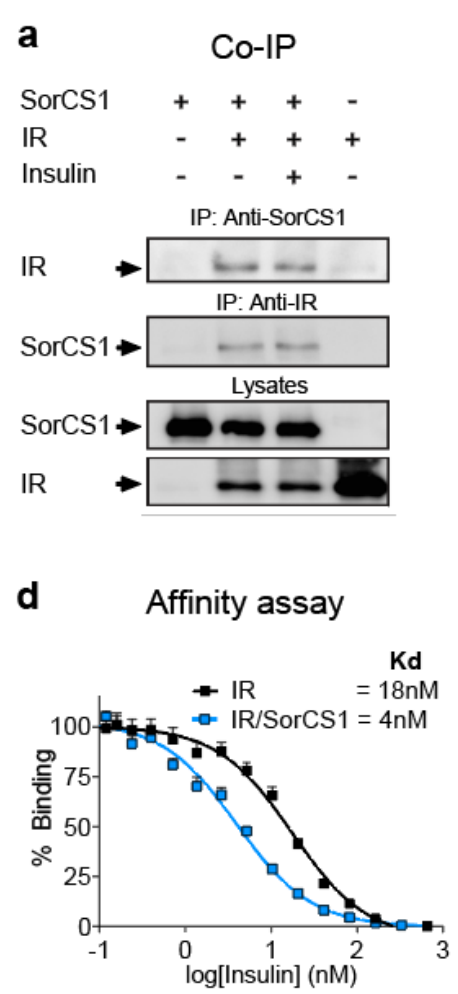

b

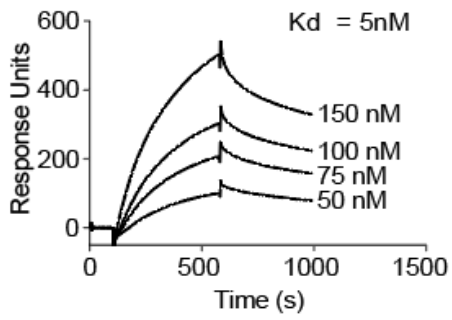

e

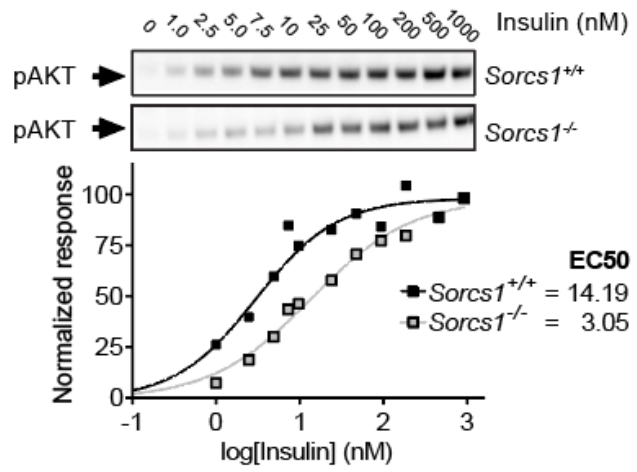

c Sol-SorCS1 \pm insulin and IR binding

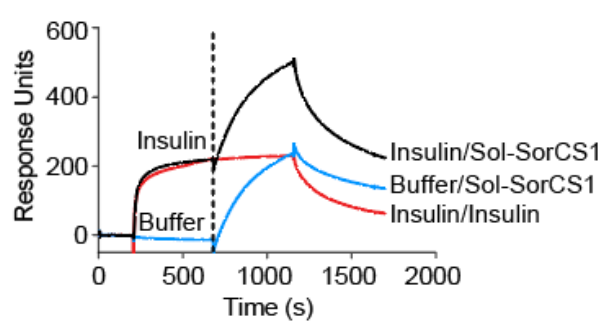

f CETSA HEK-IR

$\begin{array}{cccccccc}\operatorname{Temp}\left({ }^{\circ} \mathrm{C}\right) & 42 & 45 & 48 & 51 & 54 & 57 & 60\end{array}$ Ctrl/SorCS1 - +
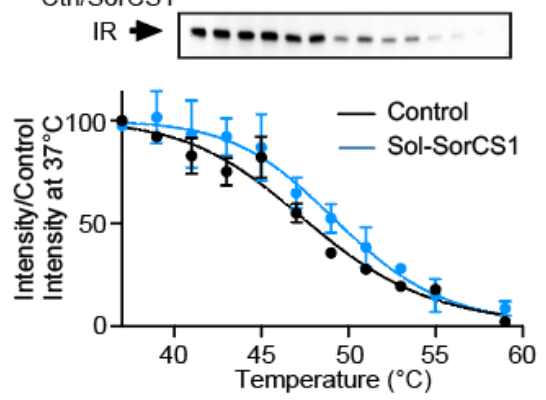

208 Figure 3: SorCS1 and IR physically interact.

a, Co-immunoprecipitation between SorCS1 and proIR/IR. The interaction is independent of the presence of saturating concentrations of insulin $(100 \mathrm{nM})$. b, SPR analysis demonstrates dose-dependent 211 binding of sol-SorCS1 to IR. c, Complex formation of SorCS1, IR, and insulin determined by SPR. At $2121200 \mathrm{sec}$, the additive signals for Sol-SorCS1 (blue) and insulin (red) equals the response units obtained 213 when IR is incubated with both proteins simultaneously (black). d, SorCS1 overexpression in HEK293 214 cells increases insulin affinity to IR. e, Reduced phosphorylation of AKT in Sorcs $1^{-/} \mathrm{C} 2 \mathrm{C} 12$ cells. f, 5 215 min. incubation with $1 \mu \mathrm{M}$ sol-SorCS1 increases thermal stability of IR from HEK-IR lysate (n=3). 
217 Figure 4
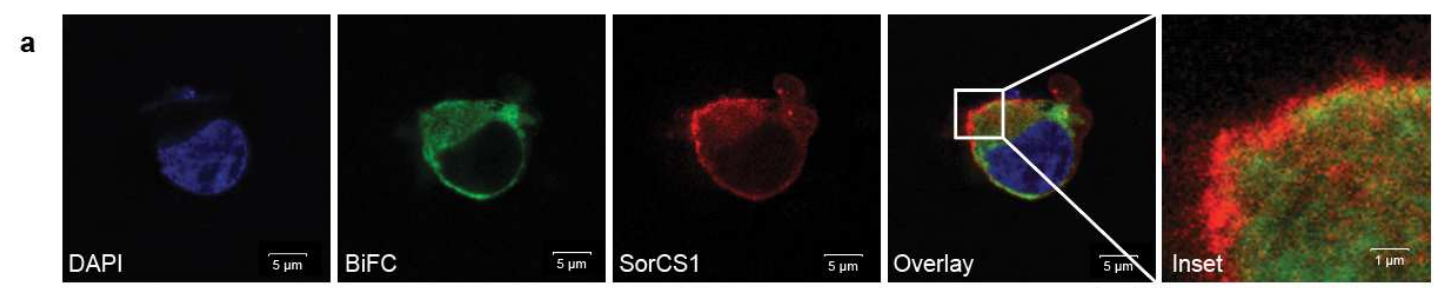

b
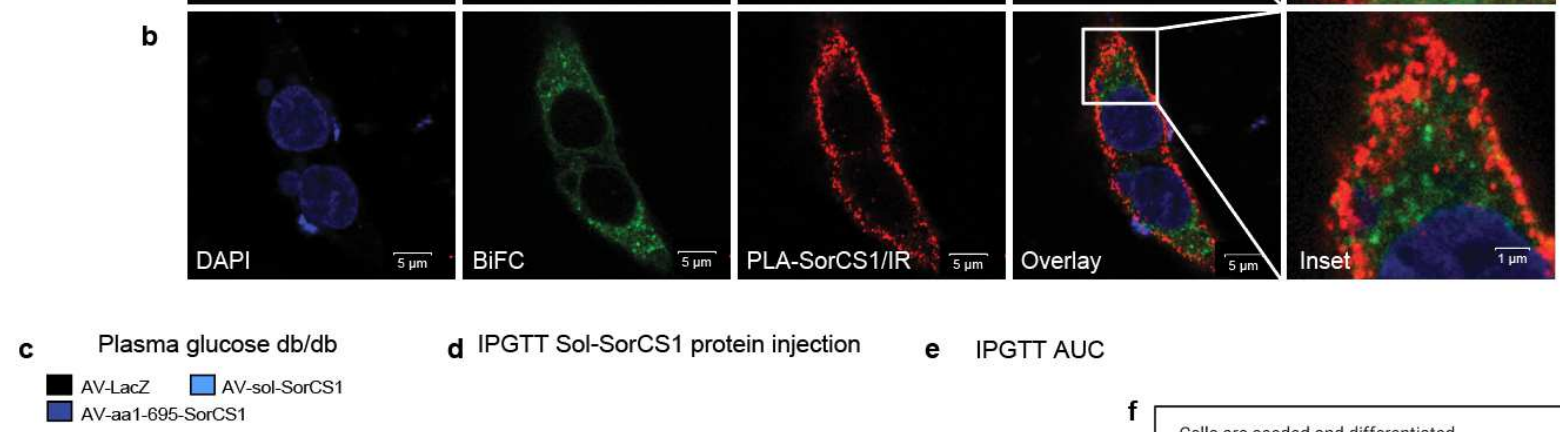

d IPGTT Sol-SorCS1 protein injection

e IPGTT AUC

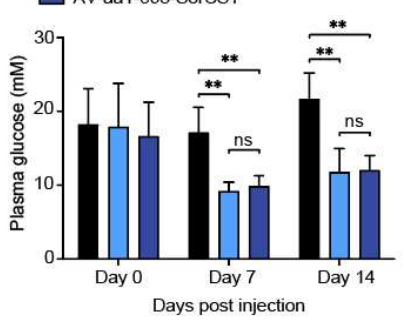

h

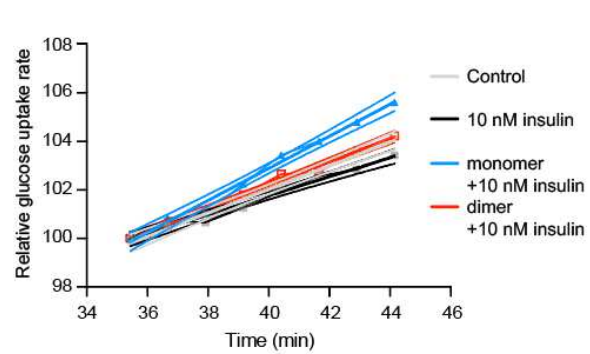

218

\section{$219 \quad$ Figure 4:}

220 A, SorCS1 dimers that are BiFC-positive (green) are confined to cytosolic compartments, whereas 221 monomeric and BiFC-negative SorCS1 ( red), is present at the cell surface (a, inset). b, in non222 permeabilized HEK293, PLA for SorCS1 and IR (red) demonstrates binding between monomeric 223 SorCS1 (BiFC-negative) and IR. c, AV-sol-SorCS1 and AV-aa1-695-SorCS1, but not AV-LacZ, reduces 224 plasma glucose in 8 weeks old $d b / d b$ mice. $\mathbf{d}$, intraperitoneal injection of recombinant monomeric, but

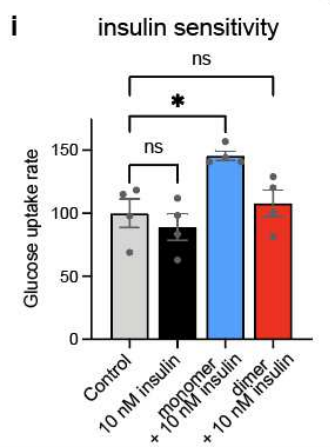

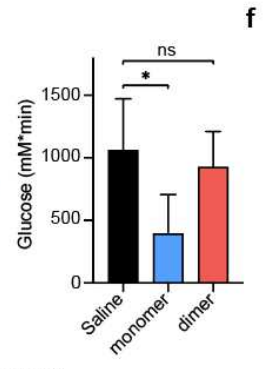
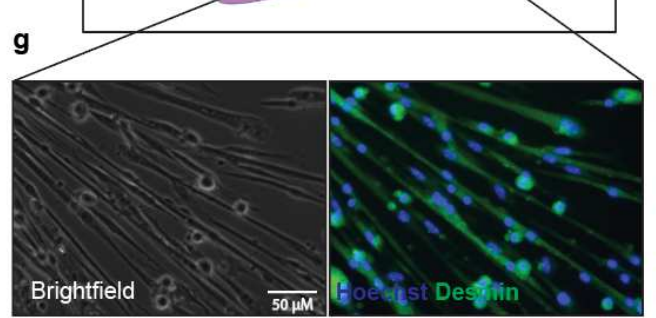
225 not dimeric, sol-SorCS1 increases glucose clearance in $d b / d b$ mice. e, AUC of the data displayed in panel 226 d. f, illustration of kinetic FDG uptake assay. $\mathbf{g}$, representative brightfield and fluorescence image of the 227 murine myotubes used for the uptake study. Hoechst in blue and Desmin in green. $\mathbf{h}$, relative FDG uptake 228 in control cells (gray), $10 \mathrm{nM}$ insulin (black), sol-SorCS1 monomer $10+\mathrm{nM}$ insulin (blue) and sol229 SorCS1 dimer $10+\mathrm{nM}$ insulin (red) $(\mathrm{n}=4)$. $\mathbf{i}$, bar chart overview of the slopes displayed in panel $\mathbf{h}$. 230 statistics are done on actual slopes. Data are presented as means $\pm \mathrm{SEM}, *=P<0.05, * *=P<0.01$. 
235 Extended data figure 1
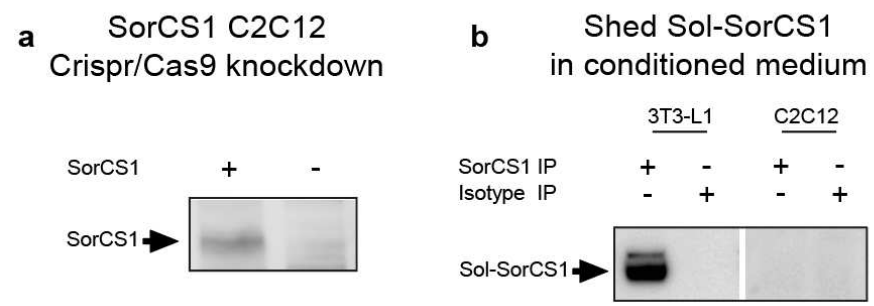

238 Extended Data Figure 1:

239 a, Through Crisp-Cas9 gene editing, we created a stable SorCS1 knockdown model of the murine C2C12 240 muscle cell line. SorCS1 protein presence was validated in the wild-type cells by western blotting using 241 a single homemade polyclonal antibody targeting murine SorCS1 (MK-S1). b, SorCS1 could not be 242 detected in serum free conditioned medium (hybridoma) from $\mathrm{C} 2 \mathrm{C} 12$ cells using two polyclonal 243 antibodies, whereas it was clearly present in conditioned medium from 3T3-L1. 
246 Extended data figure 2

247

\begin{tabular}{lll}
\multicolumn{3}{c}{ Sol-SorCS1 or IR binding } \\
\hline & Sol-SorCS1 & Insulin Receptor \\
\hline Insulin & No binding & $\mathrm{Kd}-9 \mathrm{nM}$ \\
Pro-Insulin & No binding & $\mathrm{Kd}-330 \mathrm{nM}$ \\
GLP1 & No binding & Not determined \\
Glucagon & No binding & Not determined \\
\hline
\end{tabular}

248

249 Extended Data Figure 2:

250 Table showing no binding between sol-SorCS1 and various key factors in peripheral plasma glucose 251 clearance.

252

253 
254 Extended data figure 3
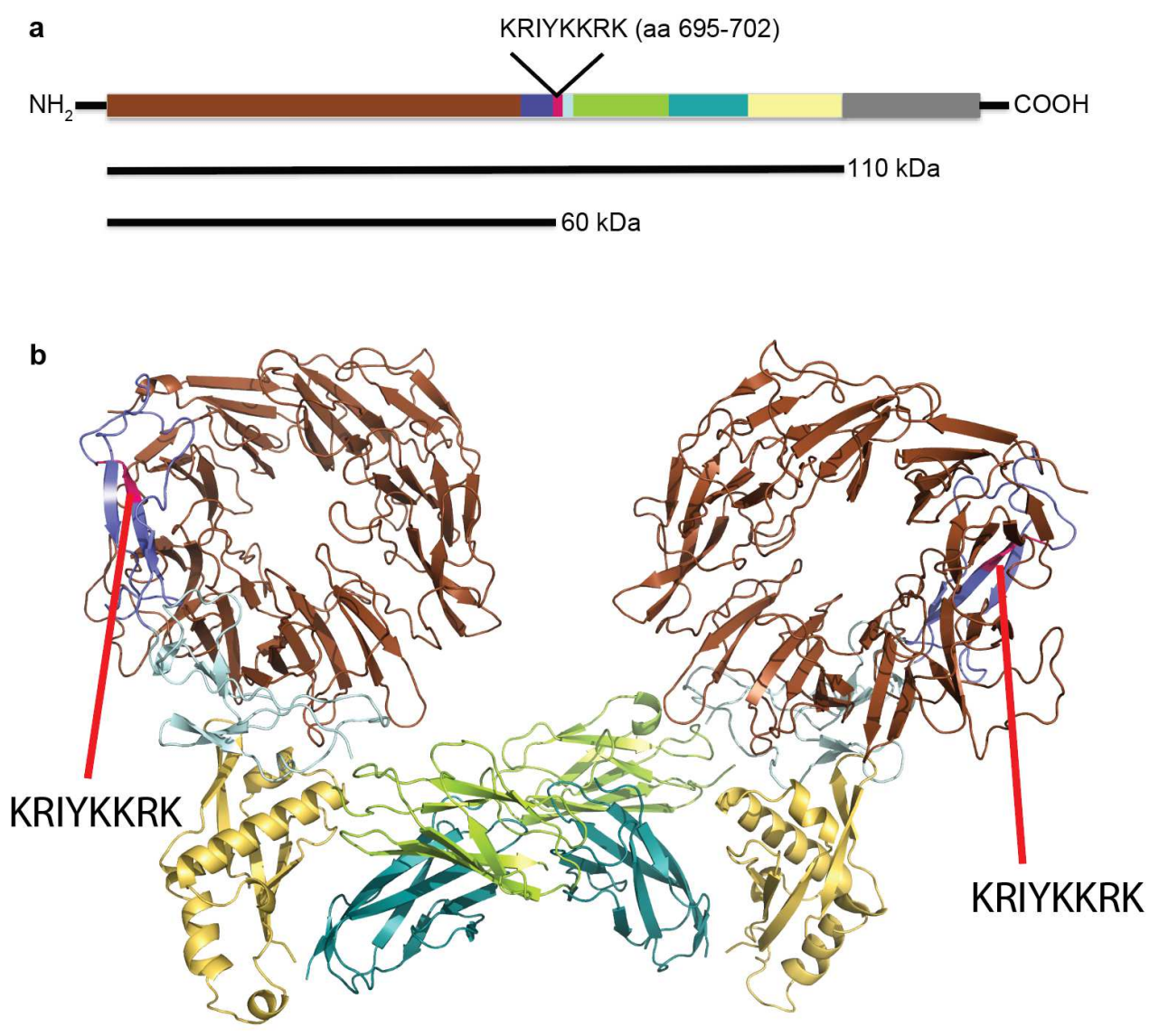

\section{Extended Data Figure 3:}

257 a, illustration of potential furin site on SorCS1 in dimer formation and the termination site of the explicit 258 monomer "aa1-695-sol-SorCS1". b, A homology model of human dimeric SorCS1 based on murine 259 SorCS2 (PDB entry 6FG9) $)^{28}$ constructed using the SwissModel service ${ }^{29}$. The individual domains were 260 manually overlaid on the single particle reconstructed envelope of monomeric SorCS1 (EMDB entry 261 EMD-3708) ${ }^{24}$. The SorCS1 dimer: Colors: $\beta$-propeller - brown, 10CCa - blue, 10CCb - lightblue, PKD1 262 - green, PKD2 - cyan, and SoMP - yellow. The position of the putative furin site (699-KKRK-702) 263 delimiting the sol 1-702-SorCS1 construct is indicated in pink. 
264 Extended data figure 4

a

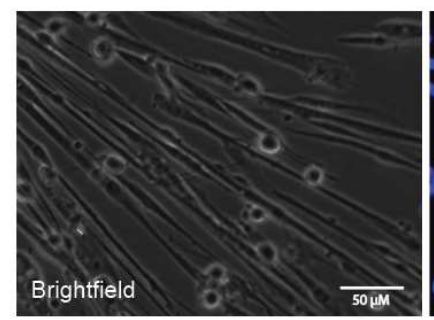

b

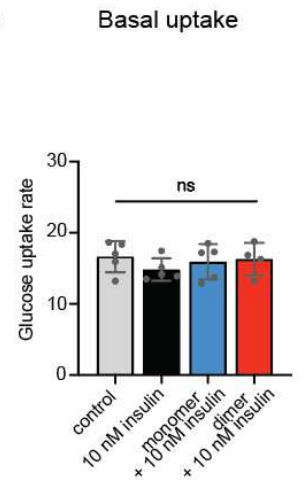

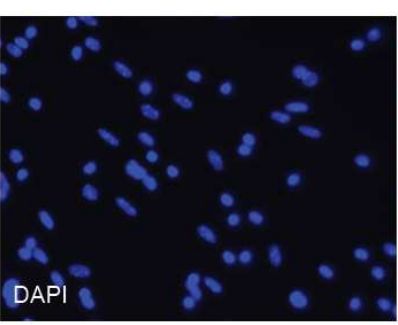
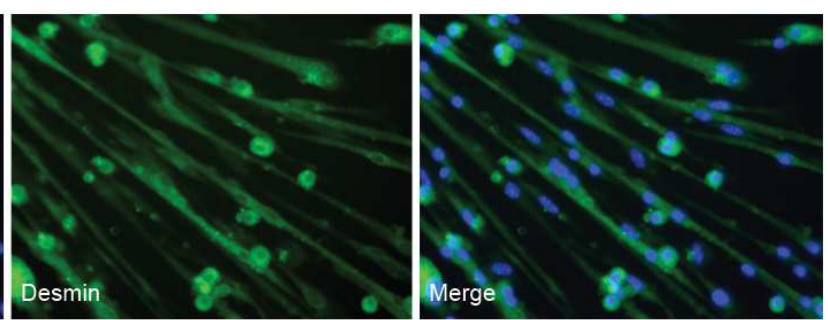

C $\quad 10 / 110 \mathrm{nM}$ insulin

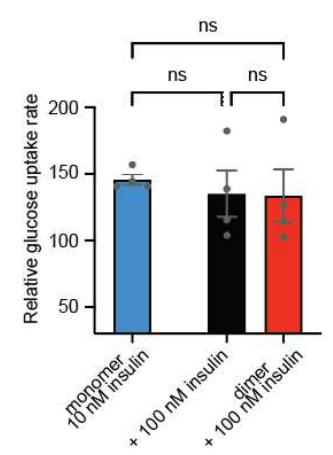

\section{Extended Data Figure 4:}

267 a, All individual panels from brightfield and fluorescence image Fig 4,h. b, Basal glucose analogue 268 uptake rate showing no difference between interventions on each study day. c, naïve myotubes and 269 myotubes pretreated with $100 \mathrm{nM}$ dimeric sol-SorCS1 increase glucose analogue uptake only when 270 stimulated with an additional $100 \mathrm{nM}$ insulin compared to $10 \mathrm{nM}$ insulin in myotubes pretreated with $271 \quad 100 \mathrm{nM}$ monomeric sol-SorCS1. 
272 Extended data figure 5

273

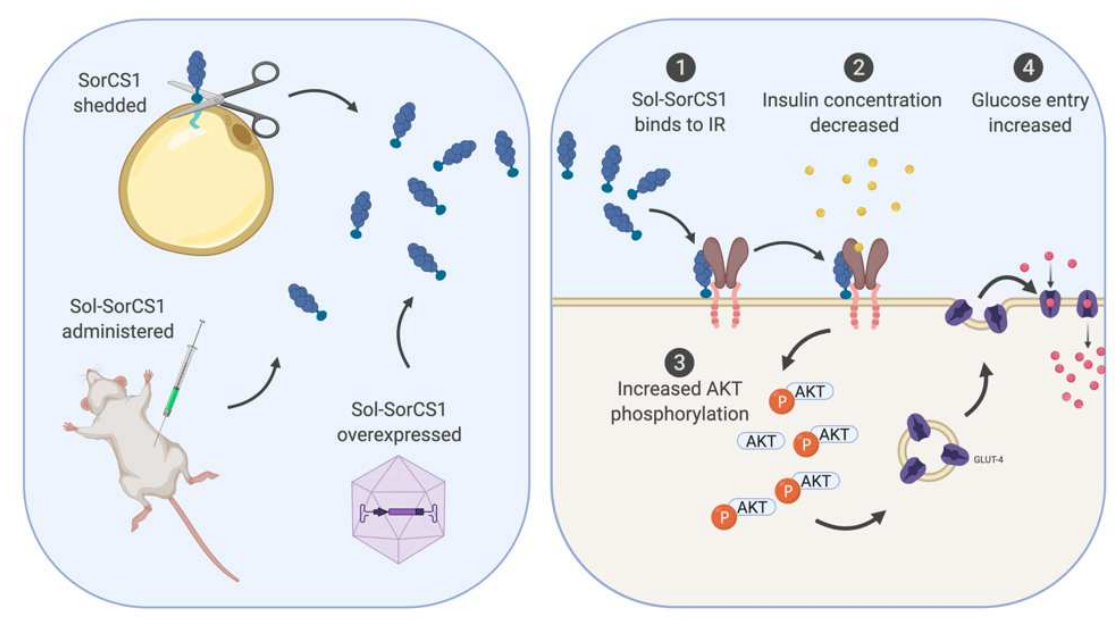

274 Extended Data Figure 5: An illustrated summery of mode of action of sol-SorCS1.

275 The illustration shows how shedding, injection or overexpression of sol-SorCS1 leads to increased 276 glucose uptake through increased insulin receptor signaling while circulating insulin concentrations 277 decrease implying increased receptor sensitivity. 
279

280

281

282

283

284

285

286

287

288

289

290

291

292

293

294

295

296

297

298

299

300

301

302

303

304

305

306

307

308

309

310

311

312

313

314

315

316

317

318

319

320

1. Shanik, M.H., et al. Insulin resistance and hyperinsulinemia: is hyperinsulinemia the cart or the horse? Diabetes Care 31 Suppl 2, S262-268 (2008).

2. Warram, J.H., Martin, B.C., Krolewski, A.S., Soeldner, J.S. \& Kahn, C.R. Slow glucose removal rate and hyperinsulinemia precede the development of type II diabetes in the offspring of diabetic parents. Ann Intern Med 113, 909-915 (1990).

3. Boucher, J., Kleinridders, A. \& Kahn, C.R. Insulin Receptor Signaling in Normal and InsulinResistant States. Cold Spring Harbor Perspectives in Biology 6, a009191-a009191 (2014).

4. Kim, S.J., et al. mTOR complex 2 regulates proper turnover of insulin receptor substrate-1 via the ubiquitin ligase subunit Fbw8. Mol Cell 48, 875-887 (2012).

5. Tremblay, F., et al. Identification of IRS-1 Ser-1101 as a target of S6K1 in nutrient- and obesity-induced insulin resistance. Proc Natl Acad Sci U S A 104, 14056-14061 (2007).

6. Blagosklonny, M.V. TOR-centric view on insulin resistance and diabetic complications: perspective for endocrinologists and gerontologists. Cell Death Dis 4, e964 (2013).

7. Willnow, T.E., Petersen, C.M. \& Nykjaer, A. VPS10P-domain receptors - regulators of neuronal viability and function. Nat Rev Neurosci 9, 899-909 (2008).

8. Willnow, T.E., Kjolby, M. \& Nykjaer, A. Sortilins: new players in lipoprotein metabolism. Curr Opin Lipidol 22, 79-85 (2011).

9. Kjolby, M., et al. Sort1, encoded by the cardiovascular risk locus 1p13.3, is a regulator of hepatic lipoprotein export. Cell Metab 12, 213-223 (2010).

10. Goettsch, C., Kjolby, M. \& Aikawa, E. Sortilin and Its Multiple Roles in Cardiovascular and Metabolic Diseases. Arterioscler Thromb Vasc Biol 38, 19-25 (2018).

11. He, Y., Fang, Z. \& Yu, G. Sortilin-related VPS10 domain containing receptor 1 and Alzheimer's disease-associated allelic variations preferentially exist in female or type 2 diabetes mellitus patients in southern Han Chinese. Psychogeriatrics 12, 215-225 (2012).

12. Paterson, A.D., et al. A genome-wide association study identifies a novel major locus for glycemic control in type 1 diabetes, as measured by both A1C and glucose. Diabetes 59, 539549 (2010).

13. Goodarzi, M.O., et al. SORCS1: a novel human type 2 diabetes susceptibility gene suggested by the mouse. Diabetes 56, 1922-1929 (2007).

14. Granhall, C., Park, H.B., Fakhrai-Rad, H. \& Luthman, H. High-resolution quantitative trait locus analysis reveals multiple diabetes susceptibility loci mapped to intervals $<800 \mathrm{~kb}$ in the species-conserved Niddmli of the GK rat. Genetics 174, 1565-1572 (2006).

15. Clee, S.M., et al. Positional cloning of Sorcs1, a type 2 diabetes quantitative trait locus. Nat Genet 38, 688-693 (2006).

16. Kebede, M.A., et al. SORCS1 is necessary for normal insulin secretory granule biogenesis in metabolically stressed beta cells. J Clin Invest 124, 4240-4256 (2014).

17. Hermey, G., Sjogaard, S.S., Petersen, C.M., Nykjaer, A. \& Gliemann, J. Tumour necrosis factor alpha-converting enzyme mediates ectodomain shedding of Vps10p-domain receptor family members. Biochem J 395, 285-293 (2006).

18. Hermey, G., et al. Characterization of sorCS1, an alternatively spliced receptor with completely different cytoplasmic domains that mediate different trafficking in cells. $J$ Biol Chem 278, 7390-7396 (2003). 
19. Graae, A.S., et al. ADAMTS9 Regulates Skeletal Muscle Insulin Sensitivity Through

20. Ayala, J.E., et al. Standard operating procedures for describing and performing metabolic tests of glucose homeostasis in mice. Dis Model Mech 3, 525-534 (2010).

21. Vaegter, C.B., et al. Sortilin associates with Trk receptors to enhance anterograde transport and neurotrophin signaling. Nat Neurosci 14, 54-61 (2011).

22. Glerup, S., et al. SorCS2 is required for BDNF-dependent plasticity in the hippocampus. Mol Psychiatry 21, 1740-1751 (2016).

23. Martinez Molina, D., et al. Monitoring drug target engagement in cells and tissues using the cellular thermal shift assay. Science 341, 84-87 (2013).

24. Januliene, D., et al. Hidden Twins: SorCS Neuroreceptors Form Stable Dimers. J Mol Biol 429, 2907-2917 (2017).

25. Kerppola, T.K. Design and implementation of bimolecular fluorescence complementation (BiFC) assays for the visualization of protein interactions in living cells. Nat Protoc 1, 12781286 (2006).

26. Nakayama, K. Furin: a mammalian subtilisin/Kex2p-like endoprotease involved in processing of a wide variety of precursor proteins. Biochem J 327 ( Pt 3), 625-635 (1997).

27. Breining, P., et al. Assessment of changes in real time net glucose uptake in adherent cell models upon acute stimulation. Bio-protocol. bio-protocol.org/prep1478. (2021).

28. Leloup, N., Chataigner, L.M.P. \& Janssen, B.J.C. Structural insights into SorCS2-Nerve Growth Factor complex formation. Nat Commun 9, 2979 (2018).

29. Waterhouse, A., et al. SWISS-MODEL: homology modelling of protein structures and complexes. Nucleic Acids Res 46, W296-W303 (2018). 


\section{Supplementary Files}

This is a list of supplementary files associated with this preprint. Click to download.

- MethodsSorCS1diabetes.pdf 